\title{
7.3 Successful use of anakinra, a soluble IL-I receptor antagonist, in pediatric rheumatic diseases associated macrophage activation syndrome/reactive hemophagocytic lymphohistiocytosis
} PM Miettunen*, A Jayanthan and A Narendran

Address: University of Calgary, Calgary, Canada

* Corresponding author from $15^{\text {th }}$ Paediatric Rheumatology European Society (PreS) Congress

London, UK. 14-17 September 2008

Published: 15 September 2008

Pediatric Rheumatology 2008, 6(SuppI I):SI3 doi:I0.II86/I546-0096-6-SI-SI3

This abstract is available from: http://www.ped-rheum.com/content/6/SI/SI3

(c) 2008 Miettunen et al; licensee BioMed Central Ltd.

\section{Background}

Increased interleukin 1 (IL-1) production characterizes macrophage activation syndrome/reactive hemophagocytic lymphohistiocytosis (MAS/rHLH), a potentially lethal complication of pediatric rheumatic diseases. Standard treatment (corticosteroids, cyclosporine, +/IVIg) is not always effective.

\section{Objective}

To test effectiveness of anakinra, a soluble IL-1 receptor antagonist, in pediatric rheumatology patients who failed to respond to standard MAS/rHLH therapy.

\section{Methods}

6 pediatric rheumatology patients (3 F:3 M); mean (range) age $8.14(0.5-13.3)$ years, with MAS/rHLH were enrolled (SoJIA $n=4$; Churg Strauss vasculitis $n=1$; and infant onset ANCA +ve pulmonary renal syndrome $n=1$ ). The infant patient was moribund in ICU. Histiocytosis society's 2004 criteria (HLH2004), including a T-cell activation marker, soluble IL-2 receptor (sIL2r), were used to confirm MAS/rHLH. Subcutaneous anakinra $(2 \mathrm{mg} / \mathrm{kg} /$ day) was added to existing therapy (high dose IV Methylprednisolone $n=5 / 6$; IVIg $n=6 / 6$, and cyclosporine $n=$ 5/6). HLH2004 specified clinical and laboratory data were collected pre, 48 hours, and 2 weeks after initiation of anakinra; including sIL2 $r$ in $3 / 6$ patients.

\section{Results}

All patients defrevesced within 24 hours of first anakinra dose, and ventilatory and dialysis support was discontinued within 96 hours in the ICU patient. All patients recovered from MAS/rHLH by 2 weeks; $5 / 6$ pts discontinued corticosteroids by 5 weeks. Abnormally elevated baseline sIL2r resolved by 48 hours post first dose of anakinra in all 3 patients tested.

\section{Conclusion}

1) Anakinra, in combination with corticosteroids, IVIG,+ / - cyclosporine, was effective in controlling MAS/rHLH in all patients, allowing rapid discontinuation of corticosteroids in $5 / 6$ patients.

2) Elevated baseline sIL2r level normalized rapidly following anakinra, suggesting resolution of abnormal T-cell activation. 